\title{
Biophysiologic Considerations in Cryoablation: A Practical Mechanistic Molecular Review
}

\author{
Michael Maccini, David Sehrt, Alexandre Pompeo, Felipe A. Chicoli, Wilson R. Molina, Fer- \\ nando J. Kim
}

Denver Health Medical Center, Division of Urology, Tony Grampsas Cancer Center, University of Colorado Health Science Center

\section{BRIEF HISTORY OF CRYOTHERAPY}

\begin{abstract}
Cryotherapy techniques date back as far as the mid-1800s, when James Arnott demonstrated the effectiveness of salt/ice mixtures in palliation of breast, uterine, and skin cancers. Subsequent advances saw the use of liquid air and solid carbon dioxide in the treatment of various conditions, particularly benign dermatologic lesions (1). Cooper and Lee introduced the first automated cryosurgical apparatus cooled by circulating liquid nitrogen in 1961 and initially used it for treating neuromuscular disorders (2). Liquid nitrogen probes were soon being used in the treatment of benign prostatic hypertrophy and prostate cancer, though complications were quite common, resulting in the procedures falling out of favor until the 1990s, when intraoperative ultrasound techniques were developed, allowing more accurate monitoring of the freezing process (1). The advent of "third-generation" argon and helium gas probes in 2000 and preoperative computer thermal mapping techniques have allowed even more precise placement, temperature control, and further reduction in post-procedural morbidity (3). Cryosurgical techniques are currently used to treat a wide variety of conditions, but significant urologic indications include treatment of low and intermediate risk prostate cancer and renal cell carcinoma $<4$ $\mathrm{cm}$ in diameter.
\end{abstract}

Key words: cryosurgery; prostatic neoplasms; kidney neoplasms

Int Braz J Urol. 2011; 37: 693-696

\section{MECHANISMS OF CELLULAR DESTRUCTION}

The cryosurgical site is characterized by two zones a central zone of total coagulative necrosis, and a peripheral zone characterized by varying degrees of cellular death and injury. The mechanisms by which acute, direct cellular death occurs in the central zone are quite well-established. The two mechanisms involved include intracellular ice crystal formation resulting in mechanical trauma, and cellular dehydration with associated osmotic damage. Subsequent cell death is mediated by ischemia and apoptosis. These mechanisms of cell death are summarized in Figure-1.

Because water diffusion through the cellular membrane is rate-dependent, rapid cooling of tissue (near the cryoablation probe) results in intracellular ice crystal formation, as water cannot leave the cell fast enough to equilibrate the intracellular and extracellular compartments (4). Intracellular ice crystal formation results in direct mechanical trauma to the plasma membrane and organelles and is lethal (4-7). Rubinsky demonstrated dramatically increased rates of cell death at lower temperatures in ND-1 prostate cancer cells at a cooling rate of 25 degrees Celsius/ min versus both 1 degree/min and 5 degrees/min (4).

Extracellular ice crystal formation occurs below -15 degrees Celsius during slow freezing (regions farther from the probe) and effectively removes water from the space surrounding cells. This creates an osmotic gradient which draws water out of the cells, stressing cell membranes and organelles and increasing intracellular electrolyte concentra- 


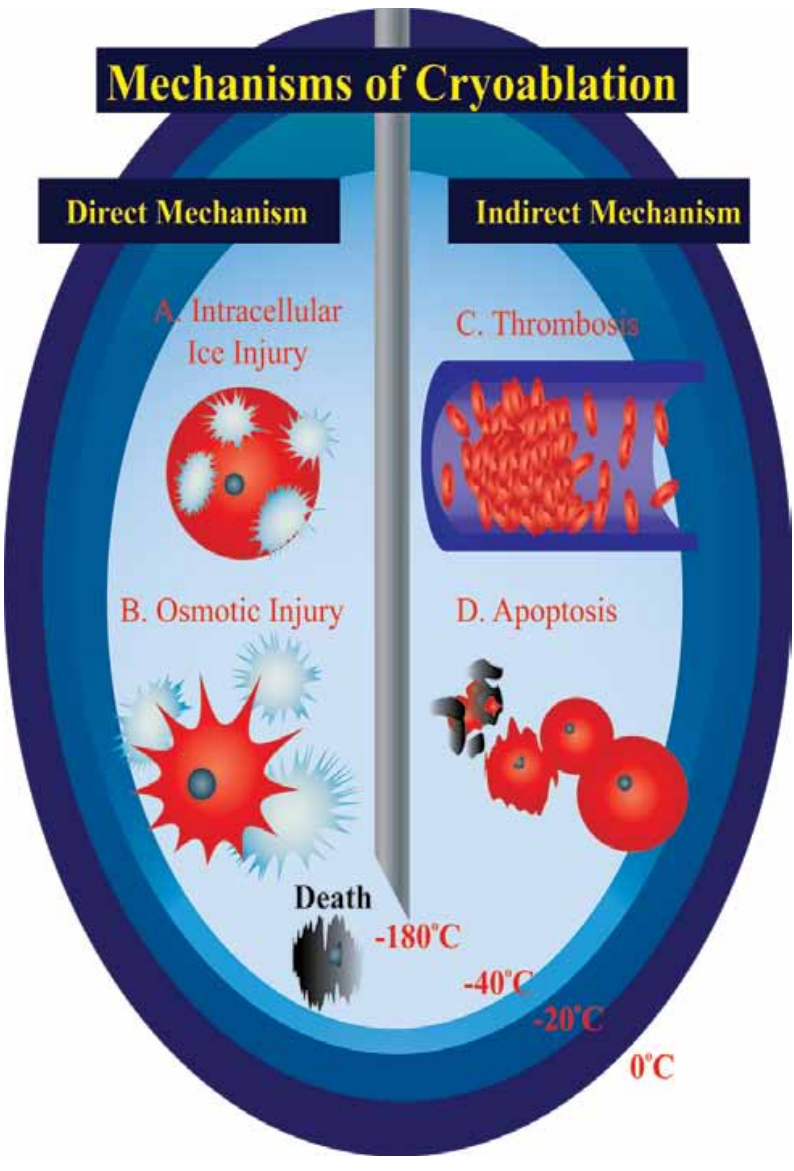

Figure 1 - A) Closest to the cryoablation probe, rapid cooling results in intracellular ice formation, directly damaging cells and resulting in immediate cell death. B) In regions farther from the probe, cooling is slower, resulting in extracellular ice formation, which creates an osmotic gradient resulting in dehydration and subsequent osmotic damage to cells, in addition to mechanical injury to membranes caused by ice crystals. C) Cryoablation also damages blood vessels, resulting in platelet activation, thrombosis, and ultimately ischemia after reperfusion. D) Lastly, cells which sustain damage-particularly damage to mitochondria (M) - not severe enough to kill them during the freezing process may undergo delayed programmed cell death (apoptosis). This may be a target for adjuvant therapies but may also be a potential mechanism for tumor resistance to cryoablation.

tions. Ice crystals outside the cell continue to grow and can cause mechanical trauma to cell membranes as well. The longer this process continues, the more likely cell death is to occur (4-7).
Slow thawing between freezing cycles results in recrystallization and further propagation of extracellular ice crystals, disrupting tissue structure (4). Thawing eventually results in a decrease in extracellular osmolarity as ice melts, which can result in an influx of water into cells, resulting in cellular swelling and bursting (5). Repeating the freeze-thaw cycle results in markedly increased cell death rates (4). This is especially important in the treatment of tumors, as there is evidence in animal models that some tumors are more resistant to damage in single freeze-thaw cycles than normal tissue, likely due to increased fibrotic tissue. Repeated freeze-thaw cycles improved local tumor control in these studies (8).

In the post-thaw period, ischemia induces further cell death in the central and peripheral zones. Endothelial damage to blood vessels results in platelet activation and thrombus formation as demonstrated in Rupp et al. histologic examination of changes in porcine post-cryoablation kidney tissue (9) leading to decreased perfusion. Additionally, Kimura et al. demonstrated decreased microvessel density and a positive correlation between hypoxia and necrosis in a mouse model of prostate cancer treated with cryosurgery (10). Ischemia induces regional hyperemia by the release of vasoactive mediators, resulting in an influx of inflammatory cells (neutrophils and macrophages). The ensuing "cleanup" process continues for weeks to months, with coagulation necrosis in the center of the surgery site and a band of neutrophils around the periphery (5).

Recent investigations have established the role of apoptosis in peripheral zone cell death. These mechanisms are not as well understood as the immediate direct injury caused by the procedure. One hypothesis is that mitochondrial damage may activate caspase cascades, resulting in programmed cell death $(5,11)$. Recognition of the role of the apoptotic pathway of cell death in cryosurgery raises questions regarding the potential for resistance to cryotherapy, particularly in the treatment of prostate cancer, as it is not possible to include the entire prostate in the central necrotic zone to ensure negative margins due to the proximity of neurovascular bundles and the rectum. This means that the peripheral portions of the prostate will be in the peripheral "injury zone" of the cryosurgical lesion, where cellular death is at 
least partially dependent on apoptosis. Given that many cancers have mutations resulting in derangements of gene-regulated cell death pathways, this could be a potential risk for recurrent disease. Baust et al. examined survival rates of in vitro prostate and colorectal cancer cell lines after a single cycle of cryoablation and found an increased cell survival rate when the cells were exposed to caspase inhibitors (12). Another study by Klossner et al. demonstrated that androgen insensitive prostate cancer cell lines showed significantly increased survival rates versus androgen sensitive cell lines after treatment (13). There are, however, also encouraging indications that adjuvant therapies may increase tumor sensitivity to cryotherapy. For example, Clarke et al. demonstrated that tumor necrosis factor-related apoptosis-inducing ligand (TRAIL) and cryoablation have a synergistic effect on a PC-3 cell line (14). This suggests that there may be cancers which are more amenable to cryosurgical treatment and cancers which are more likely to be resistant, and that there may be a role for adjuvant therapies. It should be noted, however, that these studies examined the effects of a single freeze-thaw cycle on the target cell populations, rather than multiple freeze-thaw cycles.

\section{DISCUSSION/CONCLUSIONS}

Cryosurgery has seen a resurgence in use in the past two decades, with the development of more advanced and precise equipment dramatically improving procedure safety. The mechanisms of cellular destruction and damage include both direct, immediate physical damage to cells and more delayed cell death due to local hypoxia and apoptosis. The role of apoptosis in the peripheral zone is a promising target for combination therapy, but also raises concerns due to the derangement of apoptotic pathways in many tumor cell populations, as evidenced by at least one study showing increased tumor survival after cryoablation in the presence of caspase inhibitors. If these pathways are not intact, cryoablation-mediated apoptosis may be impaired, rendering some tumors relatively resistant to cryosurgery. Further studies are needed to examine the effects of abnormal apoptotic pathways to identify potential adjuvant therapies and tumor characteris- tics which suggest the effective road to cryotherapy. In addition, using two freeze-thaw cycles in subsequent studies would more accurately simulate clinical situations.

\section{CONFLICT OF INTEREST}

None declared.

\section{REFERENCES}

1. Gage AA: History of cryosurgery. Semin Surg. Oncol. 1998; 14: 99-109.

2. Cooper IS, Lee AS: Cryostatic congelation: a system for producing a limited, controlled region of cooling or freezing of biologic tissues. J Nerv Ment Dis. 1961; 133: 259-63.

3. Babaian RJ, Donnelly B, Bahn D, Baust JG, Dineen $\mathrm{M}$, Ellis D, et al.: Best practice statement on cryosurgery for the treatment of localized prostate cancer. J Urol. 2008; 180: 1993-2004.

4. Rubinsky B: Cryosurgery. Annu Rev Biomed Eng. 2000; 2: 157-87.

5. Erinjeri JP, Clark TW: Cryoablation: mechanism of action and devices. J Vasc Interv Radiol. 2010; 21(8 Suppl): S187-91.

6. Baust JG, Gage AA: The molecular basis of cryosurgery. BJU Int. 2005; 95: 1187-91.

7. Gage AA, Baust J: Mechanisms of tissue injury in cryosurgery. Cryobiology. 1998; 37: 171-86.

8. Weber S, Lee F: Cryoablation: History, Mechanism of Action, and Guidance Modalities. Tumor Ablation. Springer, New York, 2005; pp. 250-65.

9. Rupp CC, Hoffmann NE, Schmidlin FR, Swanlund DJ, Bischof JC, Coad JE: Cryosurgical changes in the porcine kidney: histologic analysis with thermal history correlation. Cryobiology. 2002; 45: 167-82.

10. Kimura M, Rabbani Z, Mouraviev V, Tsivian M, Vujaskovic Z, Satoh T, et al.: Morphology of hypoxia following cryoablation in a prostate cancer murine model: its relationship to necrosis, apoptosis and, microvessel density. Cryobiology. 2010; 61: 148-54.

11. Yang WL, Addona T, Nair DG, Qi L, Ravikumar TS: Apoptosis induced by cryo-injury in human colorectal cancer cells is associated with mitochondrial dysfunction. Int J Cancer. 2003; 103: 360-9.

12. Baust JG, Gage AA, Clarke D, Baust JM, Van Buskirk R: Cryosurgery--a putative approach to molecularbased optimization. Cryobiology. 2004; 48: 190-204. 
13. Klossner DP, Baust JM, VanBuskirk RG, Gage AA, Baust JG: Cryoablative response of prostate cancer cells is influenced by androgen receptor expression. BJU Int. 2008; 101: 1310-6.
14. Clarke DM, Robilotto AT, VanBuskirk RG, Baust JG, Gage AA, Baust JM: Targeted induction of apoptosis via TRAIL and cryoablation: a novel strategy for the treatment of prostate cancer. Prostate Cancer Prostatic Dis. 2007; 10: 175-84.

Submitted for publication:

July 25, 2011

\section{Correspondence address:}

Dr. Fernando J. Kim

Chief of Urology, DHMC

Associate Professor of Surgery/Urology, UCHSC

Accepted after revision:

Director of Minimally Invasive Urological Oncology

August 23, 2011

Tony Grampsas Cancer Center, UCHSC

FAX: + 1303 436-6572

E-mail: fernando.kim@dhha.org 